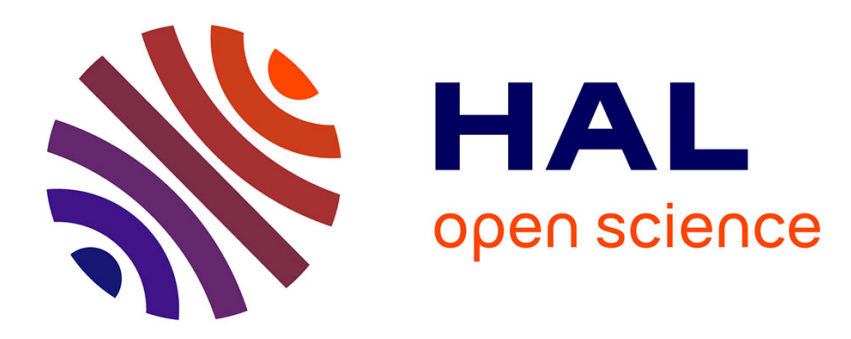

\title{
Les systèmes de tons dans les langues viet-muong
}

Michel Ferlus

\section{To cite this version:}

Michel Ferlus. Les systèmes de tons dans les langues viet-muong. Diachronica, 1998, 15 (1), pp.1-27. halshs-00922841

\section{HAL Id: halshs-00922841 \\ https://shs.hal.science/halshs-00922841}

Submitted on 31 Dec 2013

HAL is a multi-disciplinary open access archive for the deposit and dissemination of scientific research documents, whether they are published or not. The documents may come from teaching and research institutions in France or abroad, or from public or private research centers.
L'archive ouverte pluridisciplinaire HAL, est destinée au dépôt et à la diffusion de documents scientifiques de niveau recherche, publiés ou non, émanant des établissements d'enseignement et de recherche français ou étrangers, des laboratoires publics ou privés. 


\title{
LES SYSTÈMES DE TONS DANS LES LANGUES VIET-MUONG
}

\author{
Michel FERLUS \\ Centre National de la Recherche Scientifique, Paris
}

\section{Proposition}

Les langues du groupe viet-muong présentent un éventail de plusieurs types de systèmes tonals qui varient tant par le nombre des tons que par les traits phonétiques utilisés. Cette situation exemplaire permet d'illustrer la théorie de la tonogénèse en lui apportant d'utiles compléments.

\section{Les langues du groupe viet-muong}

Le groupe viet-muong est l'un des dix groupes qui composent la branche môn-khmer de la grande famille des langues austroasiatiques. Ce groupe est par ailleurs nommé "vietic" par les auteurs anglo-saxons ou encore "việt-chứt" par certains auteurs vietnamiens. Sur des bases strictement linguistiques, nous avons divisé les langues du groupe viet-muong en huit sous groupes de langues et dialectes (Ferlus 1996). Chaque sous groupe est désigné par un numéro, de 1 à 8 , et par un nom conventionnellement choisi (voir: Carte des langues et dialectes viet-muong). Note: les noms des langues utilisées sont indiqués en italique dans la classification.

1-maleng: maleng (proprement dit), malang, pakatan, mãliềng, maleng brô, kha phong (ou maleng kari).

2-arem: arem (ou cmbrau).

3-chứt: sách (ou chứt, ou salang), rục.

4-aheu: thavung, phôn soung, sô (ou sô thavung).

5-hung: pong (ou phong), toum, liha, đan lai.

6-thổ: cuối, mọn.

7-mường: mường (ou mọl, ou mọn); comporte de nombreux dialectes dont $m$. Đằm, $m$. Khói et Tân Phong; nguồn.

8-viet: viet standard écrit et ses dialectes, dial. du Centre-Nord.

Cette classification se démarque de la classification vietnamienne en quatre sous groupes (chứt, thổ, mường et việt) qui ne porte que sur les langues localisées au Vietnam (Đặng Ngiêm Vạn et al. 1986). Les mêmes termes peuvent se retrouver dans les deux classifications avec des acceptations différentes. Ainsi le sous groupe chứt de la classification vietnamienne regroupe des dia- 
lectes de nos sous groupes 1-maleng, 2-arem et 3-chứt. Pareillement, le sous groupe thổ regroupe partiellement nos 5-hung et 6-thổ. Quant au sous groupe 4-aheu, étant localisé au Laos et en Thailande, il est absent de la classification vietnamienne.

\section{Sources des données utilisées}

En dehors du vietnamien depuis longtemps bien pourvu en dictionnaires de qualité, seules trois langues du groupe viet-muong ont fait l'objet de publications utilisables dans le comparatisme, le thavung (Ferlus 1979), le mường Khói (Sokolovskaia et al. 1987) et le rục (Nguyễn Văn Lợi 1993). Les autres données utilisées sont encore inédites et ont été collectées par l'auteur lors de différents séjours ou missions de recherche sur le terrain. Avant 1970, au Laos, nous avons recueilli nous-même les premières données sur le thavung, le phôn soung et le pakatan, et malgré d'inévitables maladresses de jeunesse cela nous a permi de résoudre un problème capital de la formation du consonantisme vietnamien (Ferlus 1982). En mars-avril 1983, au Vietnam, collectes de dialectes mường en collaboration avec l'Institut de Linguistique de Hanôi. Des collègues vietnamiens ont eu l'amabilité de nous fournir des données, M Nguyễn Văn Tài sur le pong et M. Vương Lộc sur un dialecte viet du Centre-Nord parlé à Vinh. En mai-juillet 1991, au Vietnam, avec M. Nguyễn Phú Phong (CNRS / Université de Paris 7) et en collaboration avec M. Trần Trí Dõi (Université de Hanôi), collectes sur le mãliềng, le kha phong, le arem, les dialectes sách et rục et des dialectes nguồn. En octobre-novembre 1991, puis en décembre-janvier 1992-93, au Laos, en collaboration avec M. Thongpheth Kingsada (Institut des Sciences Sociales, Vientiane), collectes sur le thavung et le phôn soung, le phong et le toum, des dialectes maleng et le salang. En décembre-janvier 1993-94, puis en mars-avril 1996, au Vietnam, en collaboration avec M. Trần Trí Dõi, collectes sur des dialectes cuối et des dialectes viet du Centre-Nord.

La transcription des données, leur interprétation et leur utilisation sont entièrement de la responsabilité de l'auteur.

\section{Définition du ton}

Notre définition du ton doit être comprise dans son sens le plus large. Aux caractéristiques classiques de hauteur (pitch) et de variation (contour) nous avons rajouté les traits de constriction glottale (creakiness) et de voix soufflée (breathiness) habituellement traités comme des types de voix (voice type). 
Conventions: Nous employons ici le système de notation étymologique par les chiffres pour indiquer les classes tonales indépendamment des réalisations phonétiques.

finale sonore f. sourde finale sourde

(v. longue) (v. brève)

\begin{tabular}{|c|c|c|c|c|c|c|}
\hline $\mathrm{a}^{1}$ & $a^{3}$ & $a^{5}$ & at7 & \multirow[t]{2}{*}{$\mathrm{O}$} & a:t7 & at9 \\
\hline$a^{2}$ & $\mathrm{a}^{4}$ & $a^{6}$ & at8 & & a:t8 & at 10 \\
\hline
\end{tabular}

- Les chiffres impairs ( $\left.\mathbf{a}^{1} \mathbf{a}^{\mathbf{3}} \mathbf{a}^{\mathbf{5}} \mathbf{a}^{7}\right)$ indiquent des tons de série haute, ou premier registre, ou registre haut, conséquents aux anciennes initiales sourdes.

- Les chiffres pairs ( $\mathbf{a}^{\mathbf{2}} \mathbf{a}^{4} \mathbf{a}^{\mathbf{6}} \mathbf{a}^{\mathbf{8}}$ ) indiquent des tons de série basse, ou second registre, ou registre bas, conséquents aux anciennes initiales sonores devenues sourdes.

- Les tons $\mathbf{a}^{\mathbf{1}} \mathbf{a}^{\mathbf{3}} \mathbf{a}^{\mathbf{5}}$ et $\mathbf{a}^{\mathbf{2}} \mathbf{a}^{\mathbf{4}} \mathbf{a}^{\mathbf{6}}$ apparaissent dans les syllabes en finale ( coda) sonore (voyelle, semi-voyelle, liquide, nasale).

- Les tons $\mathbf{a}^{7}$ et $\mathbf{a}^{8}$ apparaissent dans les syllabes en finale sourde (occlusive sourde, ici symbolisée par t). Si les tons dépendent de la longueur vocalique on distinguera $\mathrm{a}^{7} \mathrm{a}^{8}$ (en voyelle longue) de $\mathrm{a}^{9} \mathrm{a}^{10}$ (en voyelle brève).

- L'accent grave [à] indique le caractère soufflé de la voyelle.

- Les réalisations phonétiques des tons, lorsqu'elles sont précisées, sont rendues par un nombre de deux ou trois chiffres entre des crochets, indiquant la hauteur et l'inflexion. Le niveau le plus bas est indiqué par $\mathrm{a}\left[{ }^{1}\right]$ et le plus haut par $\mathrm{a}\left[{ }^{5}\right]$, le symbole $\mathrm{a}\left[{ }^{5}\right]$, éventuellement $\mathrm{a}\left[{ }^{2}\right]$, précise le degré de la constriction glottale. Sur le terrain on peut observer en général une certaine diversité dans une même aire dialectale. Les réalisations phonétiques rapportées dans cette étude ne sont valables que pour le point d'enquête et ne prétendent pas être la norme de la langue ou du dialecte en question.

\section{Généralités sur la tonogénèse en viet-muong}

La formation des systèmes de tons dans les langues viet-muong (VM), comme dans la plupart des langues de l'Asie Orientale, résulte de deux phénomènes distincts.

4.1 Formation de tons à la suite de la chute des deux articulations laryngales en finale de syllabe: d'une part l'occlusive glottale $(-2)$ ou la constriction glottale $\left(-^{2}\right)$, d'autre part la spirante finale $(-h)$. Plus généralement, d'autres unités finales peuvent participer à cette tonogénèse à condition qu'elles se transforment dans l'une ou l'autre de ces articulations. Dans de nombreuses 
langues de l'Asie Orientale (dialectes chinois, tibéto-birman, miao-yao) les occlusives finales peuvent donner des tons par le biais de l'évolution $\mathrm{p} \mathrm{t}$ $\mathrm{k}>$ ?. Pareillement, la sifflante finale peut donner un ton par l'évolution $\mathrm{s}>\mathrm{h}$. Ce dernier cas peut s'observer en viet-muong. Pour rester strictement dans les langues du groupe viet-muong qui nous occupent ici, les éléments de base de la tonogénèse à retenir sont la constriction $\left(-^{2}\right)$ et la spirante $(-h)$, quelle que soit leur origine. La spirante, étant une vraie consonne finale, ne peut apparaître qu'après une voyelle, tandis que la constriction peut se combiner avec toutes les syllabes en finale sonore (voyelle, semi-voyelle, liquide, nasale). Ces deux éléments et leur absence en finale sonore forment la triade a $\mathrm{a}^{\mathrm{P}} \mathrm{ah}$, base sur laquelle se développera le premier stade de la tonogénèse.

Dans les langues VM qui ont un système tonal de base à 6 tons dans les syllabes en finale sonore il y a eu les développements suivants:

$$
\begin{aligned}
& \mathrm{a}>\mathrm{a}^{1} \text { et } \mathrm{a}^{2} \\
& \mathrm{a}^{2}>\mathrm{a}^{3} \text { et } \mathrm{a}^{4} \\
& \mathrm{ah}>\mathrm{a}^{5} \text { et } \mathrm{a}^{6}
\end{aligned}
$$

Dans les syllabes en finale sourde:

$$
\begin{aligned}
& \text { at }>\text { at7 et at } 8 \\
& \text { ou a:t }>\text { a:t7 et a:t8 (voyelle longue) } \\
& \text { at }>\text { at } 9 \text { et at } 10 \text { (voyelle brève) }
\end{aligned}
$$

Dans ce dernier cas il n'y a pas d'opposition pertinente entre les tons des longues et des brèves d'une même série, la double notation servant à distinguer des réalisations phonétiques différentes. Il faut cependant noter que dans certaines langues, autres que VM, les rimes peuvent évoluer différemment selon la longueur vocalique et dans ce cas une notation étymologique distincte se justifie pleinement.

Le problème du rapport entre la constriction, seule reconstructible en $\mathrm{VM}$, et l'occlusive glottale finale relève de considérations plus générales sur les rapports entre le VM et les autres groupes linguistiques de la famille austroasiatique et il n'y a pas lieu d'en débattre dans cette brève étude.

4.2 Formation de tons à la suite de la confusion des séries d'initiales. Si deux séries se confondent, ce qui est le cas en VM, il y a bipartition du nombre des tons issus du processus précédent. Néanmoins, des tripartitions sont observables dans de nombreuses langues d'Asie. Dans les langues VM, les tons des syllabes en finale sonore et les tons des syllabes en finale sourde forment deux sous-systèmes qui restent distincts sans interférer.

La succession des deux processus est assez claire pour les langues VM du Nord (viet, mường et thổ) dans lesquelles la formation des trois tons pri- 
mordiaux semble avoir précédé la bipartition, d'où les systèmes de base à 6 tons. Dans les langues conservatrices du Sud, la bipartition s'est produite avant la transformation de $\mathrm{ah}$, d'où les systèmes de base à 4 tons. Mais, dans ce cas de figure, on peut se demander où est la frontière entre une constriction glottale et un ton constrictif? Quand peut-on dire que la constriction est devenue mélodique? Des langues comme le sách/rục ou le thavung étaient probablement tonales avant la bipartition. Mais, une langue comme le arem, qui accuse une constriction glottale assez marquée, devait être encore non tonale lors des bipartitions comme au premier stade du proto viet-muong (PVM).

André G. Haudricourt (1954) a été le premier à avancer une explication claire de la formation des tons en vietnamien. Plus récemment, Gérard Diffloth (1989) a apporté un point de vue intéressant sur l'origine austroasiatique de la constriction glottale. L'état actuel du comparatisme ne permet pas de se prononcer et, d'autre part, ces phénomènes se situant avant le PVM sortent du cadre de cette étude.

\section{Tons ou registres?}

L'opposition, entretenue par certains linguistes, entre langues à système purement tonal et langues à registres de type de voix (voice type register) est un faux problème. Ces deux types ne représentent que des étapes dans une même chaîne de changements. En un mot, un système tonal n'est qu'une des solutions possibles de la transformation d'un système à type de voix. Dans l'aire de l'Asie Orientale qui nous concerne, toute langue qui accompli la confusion de ses séries d'initiales sourdes et sonores, dévoisement des obstruantes (occlusives, fricatives) et revoisement des résonantes (spirantes, nasales, liquides, semi-voyelles) commence toujours par développer un stade à registres de type de voix caractérisé par l'opposition d'un registre /haut clair/ à un registre /bas soufflé/. En un mot, l'opposition de registres à type de voix est la première réponse à la confusion des séries d'initiales. Ces phénomènes, aujourd'hui bien cernés, ont été révélés pour la première fois à propos du khmer (Henderson 1952).

Rappelons très schématiquement, en nous limitant aux occlusives, le mode de production des traits /sourd/ et/sonore/. Si, lors du relâchement d'une occlusion supraglottique, la glotte est en position ouverte, l'air expiré passe librement et produit une sourde ( $\mathrm{p} \mathrm{t} \mathrm{k}$ ). Si la glotte est en position serrée (mais non occluse) l'air expiré traverse la glotte en vibrant et produit une sonore (b d g).

Examinons en détail les modalités du dévoisement des occlusives sonores. Dans ce processus, qui ne peut être que diachroniquement progressif, l'ouverture de la glotte commence par un léger desserrement d'une partie des 
cordes vocales, ou plutôt des parois de la glotte, laissant passer un peu d'air bruyant qui corrompt les vibrations sonores. Le phénomène en s'amplifiant se déplace vers les voyelles grâce à la continuité de sonorité tandis que les occlusives sonores perdent progressivement leur trait de sonorité en se rapprochant de plus en plus des occlusives sourdes. Lorsque le dévoisement est consommé ( $\mathrm{b} \mathrm{d} \mathrm{g}>\mathrm{p} \mathrm{t} \mathrm{k}$ ) nous sommes dans la situation des langues à registres de type de voix. Une ancienne opposition d'occlusives, basée sur le trait de sonorité, est devenue une opposition vocalique, basée sur le trait soufflé. La surconsommation de flux expiratoire, nécessaire à la réalisation de ce souffle, engendre l'apparition d'un certain nombre de traits phoniques concomitants qui participent à l'opposition /voix claire/vs /voix soufflée/ en amplifiant la pertinence du trait soufflé. On peut y observer un abaissement du larynx associé à une dilatation de la cavité supraglottique. La hauteur mélodique tend à s'abaisser tandis que les voyelles tendent à se fermer en début d'émission. Toutes ces manifestations sont dites de "série basse".

Parallèlement, les anciennes occlusives sourdes restées sourdes ( $\mathrm{p} \mathrm{t} \mathrm{k}=$ p t k) peuvent, lorsque les anciennes sonores se rapprochent, développer une certaine tension avec une élévation de la hauteur mélodique et une tendance à ouvrir les voyelles en début d'émission. Toutes ces manifestations sont dites de "série haute". Lors de ces phénomènes, les aspirées $\left(\mathrm{p}^{\mathrm{h}} \mathrm{t}^{\mathrm{h}} \mathrm{k}^{\mathrm{h}}\right)$ et les glottalisées $(6$ d) se comportent comme des sourdes.

Résumons ces possibilités d'évolutions en un seul tableau:

\begin{tabular}{|l|c|c|c|c|}
\hline $\begin{array}{l}\text { consonne initiale } \\
\text { (occlusive) }\end{array}$ & \multicolumn{4}{|c|}{ voyelle } \\
\hline sourde $=$ sourde (tendue) & claire & haut & tendu & ouverture \\
\hline sonore $>$ sourde (relâchée) & soufflée & bas & normale & fermeture \\
\hline
\end{tabular}

Le trait soufflé est nécessaire (il ne peut pas ne pas être), et le seul qui doive apparaître obligatoirement dans le processus de la tonogénèse. Tous les traits concomitants, qu'ils soient de série haute ou de série basse, sont combinables dans un même état de langue et chaque trait est variable en intensité. La grande variété des situations dans les langues de l'Asie Orientale, et de la famille austroasiatique en particulier, offre des exemples illustrant presque toutes les possibilités de combinaison. Selon les observations diachroniques, le trait soufflé est instable et tend à disparaître en laissant la place à des caractéristiques distinctives plus stables: opposition tonale ou différenciation vocalique selon les séries. 


\section{Système de base à 6 tons: vietnamien, muò̀ng}

Ce système est typiquement celui des langues et des dialectes VM septentrionaux, vietnamien et mường.

- 6 tons dans les syllabes en finale sonore (voyelle, semi-voyelle, nasale, liquide).

- 2 tons dans les syllabes en finale sourde (occlusive sourde).

- pas de voix soufflée.

- pas de modification d'aperture vocalique liée aux tons.

\begin{tabular}{|l|l|l|}
\hline$a^{1}$ & $a^{3}$ & $a^{5}$ \\
\hline$a^{2}$ & $a^{4}$ & $a^{6}$ \\
\hline
\end{tabular} \begin{tabular}{|l|l|}
\hline$a t^{7}$ \\
\hline$a t 8$ \\
\hline
\end{tabular}

Le système complet des tons du vietnamien standard est parfaitement rendu par son écriture. Dans les dialectes parlés certains réalisent les 6 tons distincts, d'autres n'en réalisent que 5 à la suite des confusions ạ-ã ou ả-ã selon les régions, la norme écrite restant la même.

viet. écrit

\begin{tabular}{|c|c|c|}
\hline $\mathrm{a}$ & á & ả \\
\hline à & $a ̣$ & $\tilde{a}$ \\
\hline
\end{tabular} \begin{tabular}{|c|}
\hline át \\
\hline ạt \\
\hline
\end{tabular}

Note: Les nombreux changements vocaliques qui caractérisent le vietnamien et le différencient du proto viet-muong et des autres langues du groupe ne sont pas liés à la formation des registres et des tons (Ferlus 1997a). Une voyelle du PVM peut donner deux, et même trois, voyelles en vietnamien. Rappelons ici, mais sans donner des explications, les principaux changements pour éclairer les correspondances lexicales.

\begin{tabular}{ccc} 
PVM & \multicolumn{2}{c}{ vietnamien } \\
& stable & modifié \\
$*_{\varepsilon:}$ & e & ia / iê- \\
$*_{0:}$ & o & ua / uô- \\
$*_{a:}$ & a & ưa / ươ-
\end{tabular}

Le système tonal de base et la diversité des réalisations dans les dialectes mường sont les mêmes qu'en vietnamien. Le mường Đằm, province de Hòa Bình, atteste 6 tons effectifs tandis que les dialectes de mường Khói (Sokolovskaia et al. 1987), province de Thanh Hóa, et de Tân Phong, province de Sơn $\mathrm{La}$, attestent 5 tons à suite à la confusion $\mathrm{a}^{4}-\mathrm{a}^{6}$ pour ne citer que ces deux exemples. 


\begin{tabular}{|c|c|c|c|c|c|}
\hline mường Đằm & {$\left[{ }^{44}\right]$} & {$[21 ’]$} & {$\left[{ }^{435}\right]$} & {$\left[{ }^{35}\right]$} & \multirow[t]{2}{*}{6 tons } \\
\hline (Hòa Bình) & {$\left[{ }^{24}\right]$} & {$[313]$} & {$\left[{ }^{11}\right]$} & {$\left[{ }^{11}\right]$} & \\
\hline mường Khói & {$\left[{ }^{43}\right]$} & {$[213]$} & {$\left[{ }^{35}\right]$} & {$[324]$} & \multirow{2}{*}{$\begin{array}{l}5 \text { tons } \\
\left(\text { conf. } \mathrm{a}^{4}-\mathrm{a}^{6}\right)\end{array}$} \\
\hline (Thanh Hóa) & {$[55]$} & \multicolumn{2}{|c|}{$\left[{ }^{32}\right]$} & {$\left[{ }^{32}\right]$} & \\
\hline Tân Phong & {$\left[{ }^{33}\right]$} & {$\left[{ }^{312}\right]$} & {$\left[{ }^{45}\right]$} & {$\left[{ }^{34}\right]$} & \multirow{2}{*}{$\begin{array}{l}5 \text { tons } \\
\left(\text { conf. } \mathrm{a}^{4}-\mathrm{a}^{6}\right)\end{array}$} \\
\hline (Sơn La) & {$\left[{ }^{54}\right]$} & \multicolumn{2}{|c|}{$\left[{ }^{55}\right]$} & {$[21]$} & \\
\hline
\end{tabular}

Les dialectes nguồn du nord du Quảng Bình sont issus d'un dialecte mường déplacé du Thanh Hóa il y a quelques siècles. Ils offrent une situation curieuse où le ton a 6 est impliqué dans deux confusions différentes selon la couche de vocabulaire, confusion $\mathrm{a}^{2}-\mathrm{a}^{6}$ dans le vocabulaire hérité, proprement nguồn, et confusion $\mathrm{a}^{4}-\mathrm{a}^{6}$ lorsque les mots au ton $\mathrm{a}^{6}$ appartiennent à la couche des anciens emprunts au vietnamien. Donnons en exemple le système du nguồn du village de Bốc Tho, district de Tân Hóa, province de Quảng Bình. Ce dialecte se remarque en outre par des évolutions consonantiques originales, ainsi les unités initiales $b d c h t r$ du viet y sont représentées par ${ }^{\mathrm{P}} \mathrm{b}$ ? t ${ }^{2} 1$.

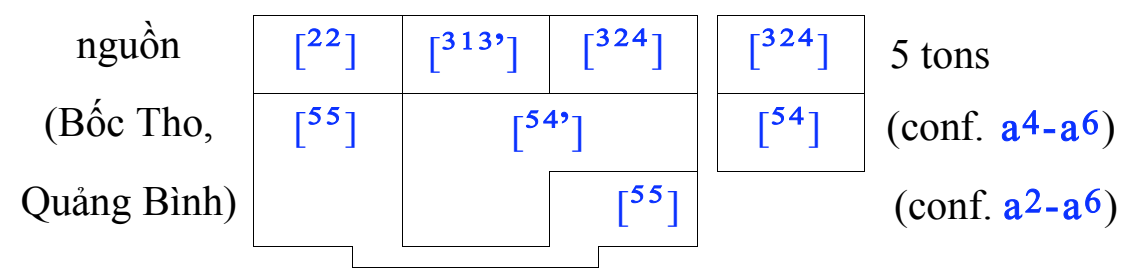

Donnons quelques exemples:

Confusion $\mathrm{a}^{2}-\mathrm{a}^{6}$ (vocabulaire hérité au ton $\mathrm{a}^{6}$ )

$\begin{array}{lcl}\text { Pa:n }\left[{ }^{55}\right] & \text { đường }\left(\mathrm{a}^{2}\right) & \text { chemin, path } \\ \text { na: }\left[{ }^{55}\right] & \text { nhà }\left(\mathrm{a}^{2}\right) & \text { maison, house } \\ \text { la:n }\left[{ }^{55}\right] & \text { lưỡi }\left(\mathrm{a}^{6}\right) & \text { langue, tongue } \\ \text { ku: }\left[{ }^{55}\right] & \text { cũ }\left(\mathrm{a}^{6}\right) & \text { ancien, ancient }\end{array}$

Confusion $\mathrm{a}^{4}-\mathrm{a}^{6}$ (vocabulaire emprunté au ton $\mathrm{a}^{6}$ ) zo:y [ [ $\left.{ }^{54}\right]$ ruộng $\left(\mathrm{a}^{4}\right)$ rizière, wet rice-field tə: $\left[^{54}\right]$ chợ $\left(\mathrm{a}^{4}\right) \quad$ marché, market

Đi: $\left[{ }^{54^{\prime}}\right] \quad$ nghĩ $\left(\mathrm{a}^{6}\right)$ penser, to think

ve: $\left[{ }^{54}\right] \quad$ vẽ $\left(\mathrm{a}^{6}\right)$ dessiner, to draw 
Pour ce qui est des tons autres que $\mathrm{a}^{6}$ les correspondances sont régulières entre le viet et le nguồn.

\section{Système de base à 6 tons: thổ}

Le cuối chăm (Ferlus 1994), pris ici comme représentant du sous groupe thổ, est localisé dans le district de Tân Kỳ, province de Nghệ An. Il présente un système tonal semblable à celui du vietnamien et du mường.

- 6 tons dans les syllabes en finale sonore.

- 2 tons dans les syllabes en finale sourde.

- pas de voix soufflée.

- modification d'aperture vocalique liée aux tons.

Donnons les réalisations tonales dans deux variantes dialectales:

\begin{tabular}{|c|c|c|c|c|}
\hline \multirow[t]{2}{*}{ cuối chăm 1} & {$\left[{ }^{45}\right]$} & {$[324]$} & {$\left[{ }^{55}\right]$} & {$\left[{ }^{34}\right]$} \\
\hline & {$\left[{ }^{43}\right]$} & {$\left[\begin{array}{ll}2 & 1\end{array}\right]$} & {$\left[{ }^{1} 1^{\prime}\right]$} & {$\left[{ }^{32}\right]$} \\
\hline \multirow[t]{2}{*}{ cuối chăm 2} & {$\left[\begin{array}{ll}1 & 1\end{array}\right]$} & {$\left[{ }^{324}\right]$} & {$\left[{ }^{55^{\prime}}\right]$} & {$\left[{ }^{23}\right]$} \\
\hline & {$\left[{ }^{54}\right]$} & {$\left[{ }^{45^{\prime}}\right]$} & {$\left[{ }^{1} 1^{\prime}\right]$} & {$\left[{ }^{32}\right]$} \\
\hline
\end{tabular}

Les tons $a^{5}$ et a ${ }^{6}$ se réalisent avec une constriction glottale bien marquée en fin d'émission vocalique. Au ton a cette constriction, plus marquée qu'en $a^{6}$, abrège sensiblement la voyelle. A la différence du vietnamien et des dialectes mường, le cuối atteste des changements d'aperture vocalique conséquents à la confusion des séries d'initiales, fermeture de *a: sous les tons de série basse et ouverture de $*_{0}$ : et ${ }^{*}{ }^{\prime}$ : sous les tons de série haute, ce qui montre clairement que cette langue est passée par le stade à registres de type de voix.

\begin{tabular}{|c|c|c|c|c|}
\hline PVM & $\mathrm{cu}$ & & & namien \\
\hline & haut & bas & & \\
\hline *a: & $a:$ & e: & $\mathrm{a}$ & ưa / ươ- \\
\hline$*_{0:}$ & $0:$ & o: & ô & \\
\hline *o: & æ: & 0: & o & ua / uô- \\
\hline
\end{tabular}

Donnons quelques exemples:

\begin{tabular}{lllll} 
PVM & \multicolumn{2}{c}{ cuối } & \multicolumn{2}{c}{ vietnamien } \\
*a: & a: & sa:t7 & tát & assécher, to dry up \\
& & da:k7 & nước & eau, water \\
& e: & je:2 & nhà & maison, house \\
& & te:n2 & đường & chemin, path
\end{tabular}




\begin{tabular}{|c|c|c|c|c|}
\hline \multirow{2}{*}{$\begin{array}{l}\text { PVM } \\
*_{0:}\end{array}$} & \multicolumn{2}{|c|}{ cuối } & \multicolumn{2}{|c|}{ vietnamien } \\
\hline & o: & 6ว:j 1 & môi & lèvres, lips \\
\hline & & ko: 5 & cổ & articulation, joint \\
\hline & o: & mo:t8 & một & un, one \\
\hline & & so:m² & tôm & crevette, shrimp \\
\hline *o: & æ: & $\mathrm{kæ:n} 1$ & con & fils, son \\
\hline & & $6 æ: j^{3}$ & muối & sel, salt \\
\hline & o: & $\begin{array}{l}\text { mo:t8 } \\
\text { ro:t8 }\end{array}$ & $\begin{array}{l}\text { mọt } \\
\text { ruột }\end{array}$ & $\begin{array}{l}\text { charançon, weevil } \\
\text { intestins, intestines }\end{array}$ \\
\hline
\end{tabular}

Le cas du cuối, et vraisemblablement des autres dialectes du sous groupe thổ, est remarquable en ce sens qu'il nous montre qu'une langue présentant un système purement tonal en n'utilisant que la mélodie, peut très bien préserver des modifications d'aperture vocalique qui sont des vestiges du premier stade à registres de type de voix. Il convient néanmoins de préciser que, dans le cas présent, seul le comparatisme permet de déceler ces changements. Ces dernières caractéristiques différencient les dialectes du sous groupe thổ du viet et du mường.

\section{Système de base à 4 tons: sách/rục}

Le sách et le rục, localisés dans le district de Tuyên Hóa, province de Quảng Bình, ne sont que deux variantes dialectales d'une même langue.

- 4 tons dans les syllabes en finale sonore.

- 2 tons dans les syllabes en finale sourde (occ. sourdes, $-\mathrm{h}$ ).

- voix soufflée / hauteur mélodique (breathiness / pitch-contour).

- modification d'aperture vocalique en série basse.

\begin{tabular}{|l|l|}
\hline $\mathrm{a}^{1}$ & $\mathrm{a}^{3}$ \\
\hline $\mathrm{a}^{2}$ & $\mathrm{a}^{4}$ \\
\hline
\end{tabular}

\begin{tabular}{|ll|}
\hline ah7 & at7 \\
\hline ah8 & at8 \\
\hline
\end{tabular}

Réalisations tonales (données personnelles):
$\mathrm{a}^{1}\left[{ }^{44}\right]$
$a^{3}\left[{ }^{45}\right]$
a7 $\left[{ }^{45}\right]$
voix claire
$\mathrm{a}^{2}\left[{ }^{11}\right]$
$\mathrm{a}^{4}\left[{ }^{11^{\prime}}\right]$
a8 $\left[{ }^{11}\right]$ voix soufflée

Le sách/rục n'a pu développer les tons attendus a $a^{5}-a^{6}$ puisque le $-\mathrm{h}$ final est conservé. Cet état est exemplaire de ce qu'on peut appeler une langue à registres de type de voix avec une opposition /voix claire/ vs /voix soufflée/ pour toutes les voyelles. La fermeture en série basse ne porte que sur les voyelles les plus ouvertes (Nguyễn Văn Lợi 1993). 

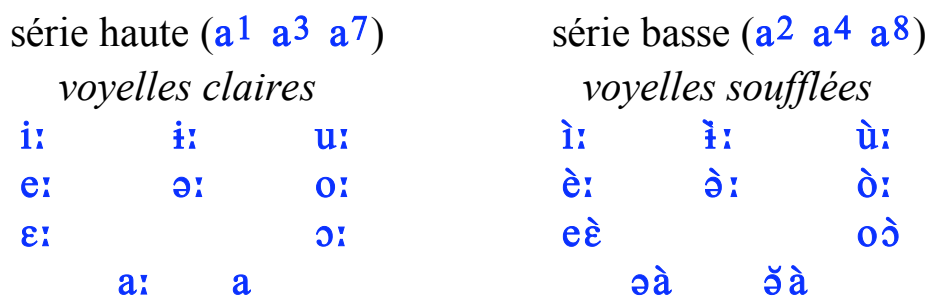

Donnons quelques exemples:

\begin{tabular}{|c|c|c|c|c|}
\hline PVM & sác & rục & viet & \\
\hline$*_{a}$ & a: & lăta:3 & đá & pierre, stone \\
\hline & əà & təày 2 & đường & chemin, path \\
\hline *a & a & dam 1 & năm & cinq, five \\
\hline & วัà & lăàn4 & lặn & plonger, to dive \\
\hline${ }^{*} \varepsilon \mathrm{z}$ & $\begin{array}{l}\varepsilon: \\
\text { èे }\end{array}$ & $\begin{array}{l}\text { je:13 } \\
\text { meغे } 4\end{array}$ & $\begin{array}{l}\text { nhẹ } \\
\text { mẹ }\end{array}$ & $\begin{array}{l}\text { léger, light in weight } \\
\text { mère, mother }\end{array}$ \\
\hline${ }^{*}{ }^{*}$ & $\begin{array}{l}\text { ०: } \\
\text { ò̀ }\end{array}$ & $\begin{array}{l}\text { alo: }{ }^{3} \\
\text { moว̀n } 2\end{array}$ & $\begin{array}{l}\text { lúa } \\
\text { mòn }\end{array}$ & $\begin{array}{l}\text { paddy, paddy } \\
\text { (sentier) battu, beater }\end{array}$ \\
\hline
\end{tabular}

Remarquons que cet état de langue ne concerne que le stock de vocabulaire proprement sách/rục. Les nombreux emprunts au nguồn et au vietnamien, parfaitement intégrés, ont introduit des voyelles non soufflées et non prédiphtonguées aux tons de série basse.

$$
\begin{array}{lll}
\text { ka:14 } & \text { cải } & \text { choux-moutarde, mustard greens } \\
\text { ya:j2 } & \text { người } & \text { Homme, Human being }
\end{array}
$$

\section{Système de base à 4 tons: thavung}

Le thavung, qui forme avec le phôn soung le sous-groupe aheu, est parlé au Laos dans le district de khamkeut, province de Bolikhamxay. Nous disposons de deux périodes d'observation, 1965-70 et 1991-93. Le système tonal de base est en gros le même qu'en sách/rục.

- 4 tons dans les syllabes en finale sonore.

- 2 tons dans les syllabes en finale sourde (occ. sourdes, -h ).

- hauteur mélodique / voix soufflée selon la voyelle.

- modification d'aperture vocalique en série basse.

\begin{tabular}{|l|l|}
\hline$a^{1}$ & $a^{3}$ \\
\hline$a^{2}$ & $a^{4}$ \\
\hline
\end{tabular} \begin{tabular}{|ll|}
\hline$a h^{7}$ & at7 \\
\hline$a h^{8}$ & at8 \\
\hline
\end{tabular}

Réalisations tonales:
$a^{1}\left[{ }^{44}\right] \quad a^{3}\left[{ }^{54}\right]$
$\mathrm{a}^{7}\left[{ }^{44}\right]$
voix claire
$\mathrm{a}^{2}\left[{ }^{11}\right] \quad \mathrm{a}^{4}\left[{ }^{11^{\prime}}\right]$
$a^{8}\left[{ }^{11}\right]$
voix soufflée dominante
(selon la voyelle) 
Comme pour le sách/rục, le thavung n'a pu développer les tons attendus $\mathrm{a}^{5}-\mathrm{a}^{6}$ puisque le $\mathrm{h}$ final est conservé (Ferlus 1979). Dans les mots en voyelle fermée (high vowel) l'opposition de hauteur mélodique est prédominante. Dans les mots en voyelle moyenne (mid high/low vowel) ou en voyelle ouverte (low vowel) l'opposition de type de voix est prédominante avec le trait /voix claire/ en série haute et le trait/voix soufflé/ associé à une fermeture vocalique par prédiphtongaison en série basse. Toutes ces caractéristiques ont été observées dans la période 1965-70. Cependant, des enquêtes plus récentes menées en 1991-93 avec des informateurs différents mais issus de la même communauté de locuteurs ont montré un état de dégradation avancée du système. Les voyelles diphtonguées de série basse se sont stabilisées en voyelles simples et les distinctions tonales, hauteur et voix, ont pratiquement disparu sans compensation, seule se maintient la constriction des anciens tons $\mathrm{a}^{3}$ et $\mathrm{a}^{4}$. Le système tend vers une opposition tonale au sens large réduite à deux termes /ton simple (non marqué)/ vs/ton constrictif/ en laissant les modifications d'aperture.

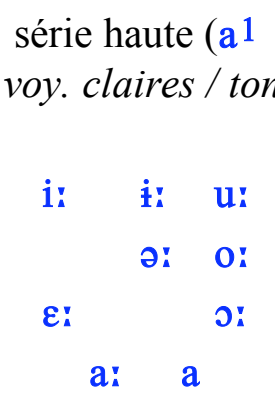

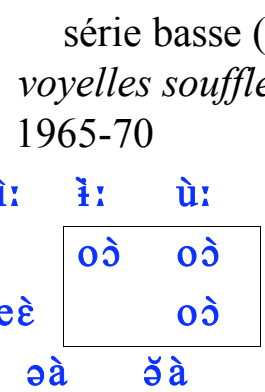

Donnons quelques exemples:

\begin{tabular}{|c|c|c|c|c|}
\hline PVM & thas & $n g(1965-70)$ & viet & \\
\hline$* a:$ & a: & $\mathrm{ka} \mathbf{i}^{3}$ & cá & poisson, fish \\
\hline & əà & cəà12 & chài & épervier (filet), casting net \\
\hline$* a$ & a & $\tan ^{3}$ & đắng & amer, sour \\
\hline & ว̆à & nə̆ àn 4 & nặng & lourd, heavy \\
\hline${ }^{*} \mathrm{e}_{\mathrm{i}}>$ ə: $_{\mathbf{I}}$ & ə: & cə:t7 & chết & mourir, to die \\
\hline & oذ̀ & voذ̀ 12 & về & rentrer, to return \\
\hline$*_{\partial:}$ & ə: & pəsə:m 3 & - & respirer, to breathe \\
\hline & ò̀ & məloj̀j2 & trời & pluie/ciel, rain/sky \\
\hline$*_{\varepsilon}:$ & $\varepsilon:$ & məhe:t7 & sét & foudre, lightning \\
\hline & èे & keغ̀n 4 & kiến & fourmi, ant \\
\hline$k^{k}$ & o: & po:n 3 & bốn & quatre, four \\
\hline & ò̀ & lò̀ $\mathrm{m}^{4}$ & trộm & voler, to rob \\
\hline : & o: & $\cos { }^{3}$ & chó & chien, $d o g$ \\
\hline & ò̀ & poj̀ $\mathrm{k}^{8}$ & buộc & attacher, to bind \\
\hline
\end{tabular}

1991-93

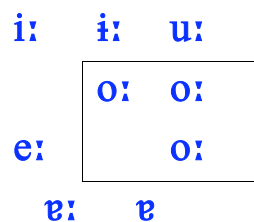


Remarquons que PVM e: s'est confondu en ə: dans les dialectes aheu (thavung, phôn soung). Chez les locuteurs thavung de Thailande du district de Song Dao, province de Sakonnakhorn, localement nommés sô et déplacés du Laos à la fin du siècle dernier, la voix soufflée et les diphtongaisons concomitantes sont mieux préservées. De plus, en série basse, la voyelle modifiée de ${ }^{*} \partial_{\text {: }}$ est restée distincte des voyelles modifiées de ${ }^{*} \mathrm{o}$ : et ${ }^{*}{ }^{2}$ : confondues.

\section{Système usé à 2 tons: maleng}

Le maleng est parlé dans une dizaine de villages dispersés dans les districts de Khamkeut, province de Bolikhamxay, et de Nhommalat, province de Khammouan, au Laos.

- 2 tons dans les syllabes en finales sonores.

- pas d'opposition de ton dans les syllabes en finales sourdes

(occ. sourde, $-\mathrm{h}$ ).

- pas de voix soufflée.

- modification d'aperture vocalique.

Cette situation est engendrée à partir d'un système à 4 tons, comme celui du sách/rục ou du thavung, dont l'opposition /voix claire/ vs /voix soufflé/ disparaît sans avoir eu le temps de se transphonologiser en une opposition tonale. Il ne subsiste plus que 2 tons, un ton non marqué sensiblement montant, résultant de la confusion de $\mathrm{a}^{1}$ et $\mathrm{a}^{2}$, et un ton constrictif (noté $\mathrm{a}^{2}$ ), ou occlus, résultant de la confusion de $\mathrm{a}^{3}$ et $\mathrm{a}^{4}$.

\begin{tabular}{|l|l|l|}
\hline $\mathrm{a}$ & $\mathrm{a}^{2}$ \\
\hline
\end{tabular} \begin{tabular}{|ll}
\hline ah at \\
\hline
\end{tabular}

Ce n'est pas à proprement parler un système tonal au sens classique du terme, on a plutôt à faire à une opposition de type vocalique /clair/ vs /constrictif/. Cependant, nous refusons l'interprétation, parfois proposée, en terme de registre de type de voix car le trait constrictif, tel qu'il est observé dans ces langues, ne se manifeste que sur une partie de la durée vocalique et n'est donc pas exactement une voix craquée (creaky voice). En maleng, la perte du souffle vocalique laisse cependant des modifications d'aperture, toutes les voyelles tendant à s'ouvrir en série haute, sauf*a: qui se ferme vers l'arrière en série basse.

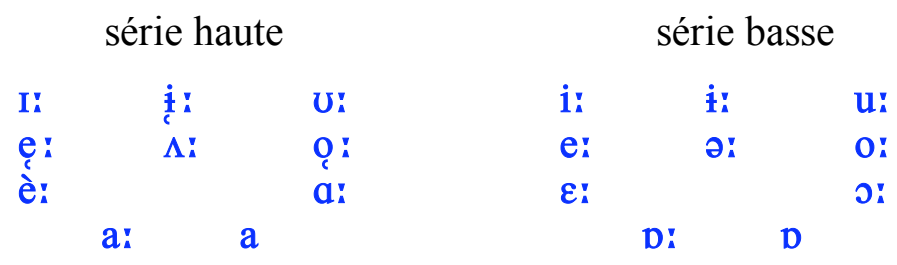


Donnons quelques exemples pour les voyelles de grande et de moyenne aperture:

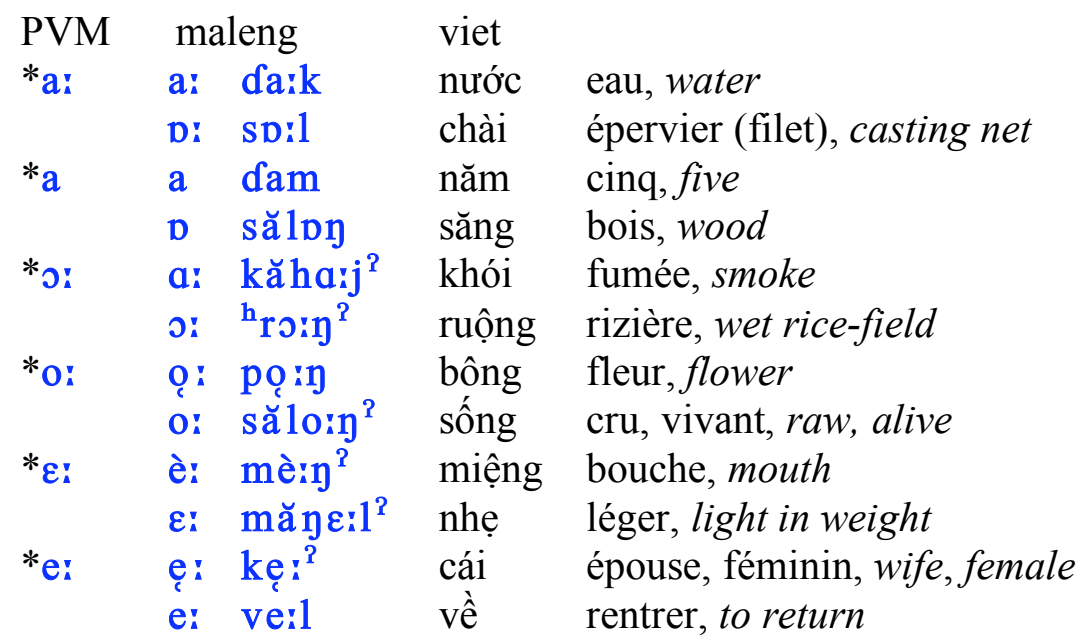

Dans la pratique ces distinctions sont difficiles à maintenir et dans certains dialectes maleng soumis à une forte influence du lao, comme le pakatan, le système vocalique tend à se simplifier et à s'identifier à celui de la langue dominante.

Le cas du vocalisme maleng rappellerait un peu celui du khmer mais, dans cette dernière langue, le trait soufflé n'a disparu qu'après que les modifications d'apertures vocaliques, suffisamment caractérisées, aient assuré les distinctions. Le maleng, trop fortement influencé par le lao, représente une ratée de la tonogénèse.

\section{Système hétérodoxe à 4 tons: pong, toum}

Le pong est parlé dans le district de Tương Dương, province de Nghệ An au Vietnam, près de la frontière lao-viet. Le toum est parlé dans le district de Viang Thong, province de Bolikhamxay au Laos.

- 4 tons dans les syllabes en finale sonore;

$-2+2$ tons dans les syllabes en finale sourde;

- pas de -h final;

- pas de voix soufflée;

- pas de modification d'aperture (pong);

- modification d'aperture (toum).

\begin{tabular}{|l|l|}
\hline $\mathrm{a}^{1}$ & $\mathrm{a}^{35}$ \\
\hline $\mathrm{a}^{2}$ & $\mathrm{a}^{46}$ \\
\hline
\end{tabular}


Par comparaison avec des langues à 6 tons de base, viet, mường ou thổ, on constate que les tons attendus $\mathrm{a}^{3}$ et $\mathrm{a}^{5}$ sont représentés par un seul ton (noté $\mathrm{a}^{35}$ ), de même pour $\mathrm{a}^{4}$ et $\mathrm{a}^{6}$ (noté $\mathrm{a}^{46}$ ). Ces réductions n'ont rien à voir avec les confusions déjà vues dans certains dialectes viet ou mường; l'explication en sera donnée plus loin. Dans les exemples suivants la classe tonale des exemples vietnamien est précisée pour une lecture plus aisée.

\begin{tabular}{|c|c|c|}
\hline $\begin{array}{l}\text { pong } \\
\text { pa:35 }\end{array}$ & $\begin{array}{l}\text { vietnamien } \\
\text { vá }\left(\mathrm{a}^{3}\right)\end{array}$ & réparer, coudre, to mend, to sew \\
\hline ba: 35 & mửa $\left(\mathrm{a}^{5}\right)$ & vomir, to vomit \\
\hline 46 & nho $\left(\mathrm{a}^{4}\right)$ & suie, soot \\
\hline & $\left(a^{6}\right)$ & fracture, fracture \\
\hline
\end{tabular}

Donnons les réalisations tonales:

pong

\begin{tabular}{|l|l|}
\hline$\left[{ }^{24}\right]$ & {$\left[{ }^{55}\right]$} \\
\hline$\left[{ }^{32}\right]$ & {$\left[^{32}\right]$} \\
\hline
\end{tabular}

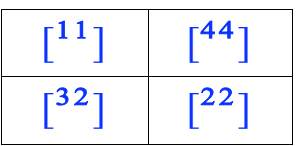

toum

\begin{tabular}{|l|l|}
\hline$\left.{ }^{55}\right]$ & {$\left[{ }^{213}\right]$} \\
\hline$\left.{ }^{11}\right]$ & {$\left[{ }^{329}\right]$} \\
\hline
\end{tabular}

\begin{tabular}{|l|l|}
\hline$\left[{ }^{13}\right]$ & {$\left[{ }^{34}\right]$} \\
\hline$\left[{ }^{53}\right]$ & {$\left[{ }^{32}\right]$} \\
\hline
\end{tabular}

Les confusions des séries d'initiales n'ont pas de conséquence sur les voyelles du pong. En revanche, en toum les voyelles d'aperture moyenne $*_{\mathrm{e}}$ : * ${ }_{\partial:} *_{0:}$ s'ouvrent en série haute en entraînant de possibles confusions avec d'autres unités. Nous ne donnerons pas d'exemples car ces changements sont conditionnés par les finales et varient énormément selon les dialectes.

\section{Systèmes hétérodoxes à 4 tons: dialectes vietnamiens du Centre-Nord}

Les dialectes dits du Centre-Nord, autrefois signalés sous le nom de 'dialectes du Haut-Annam' (Cadière 1902), se maintiennent dans une aire discontinue couvrant le sud du Nghệ An (Ferlus 1991), la bande côtière du Hà Tĩnh et quelques isolats dans le Quảng Bình. Ces dialectes ont été qualifiés d'hétérodoxes parce que leur phonétisme atypique ne peut s'expliquer par les lois de la phonétique historique qui régissent la formation du vietnamien standard et de ses dialectes. Les graphies de l'écriture actuelle, élaborée pour l'essentiel au XVIIe siècle (de Rhodes 1651), ne conviennent pas à ces dialectes du Centre-Nord.

La plupart de ces dialectes présentent un système de base à 6 tons, réduit à 5 tons effectifs par la confusion $\mathrm{a}^{4}-\mathrm{a}^{6}$ (viet: ạ-ã). Cependant ceux 
parlés au nord du Quảng Bình, dans le bassin du Sông Gianh, attestent des systèmes à 4 tons dont l'originalité réside dans les traitements des tons attendus a 5 et a 6 (viet: ả et ã), c'est-à-dire ceux qui proviennent normalement de l'ancien -h final. On y observe deux types de confusion (Nguyễn Tài Cẩn 1995:226):

- Confusions de $\mathrm{a}^{3}-\mathrm{a}^{5}$ et de $\mathrm{a}^{4}-\mathrm{a}^{6}$ respectivement: dialecte de Hướng Hóa, district de Tuyên Hóa.

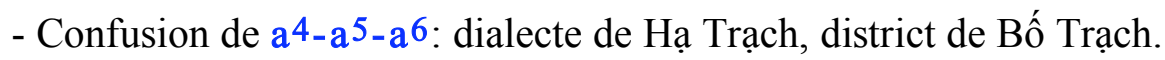

\begin{tabular}{|l|l|}
\hline $\mathrm{a}^{1}$ & $\mathrm{a}^{35}$ \\
\hline $\mathrm{a}^{2}$ & $\mathrm{a}^{46}$ \\
\hline
\end{tabular}

Hướng Hóa [Tuyên Hóa]

\begin{tabular}{|l|c|}
\hline $\mathrm{a}^{1}$ & $\mathrm{a}^{3}$ \\
\hline $\mathrm{a}^{2}$ & $\mathrm{a}^{456}$ \\
\hline
\end{tabular}

Le dialecte de Cao Lao Hạ (Ferlus 1995), district de Bố Trạch, appartiennent au deuxième type. Donnons-en les réalisations phonétiques et quelques exemples de confusions.

Cao Lao Hạ
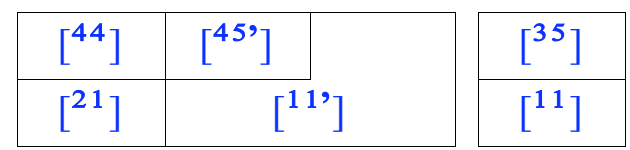

$\begin{array}{llll}\text { zo:j456 } & \text { ruộng } & \left(\mathrm{a}^{4}\right) & \text { rizière, wet rice-field } \\ \text { tlo:m456 } & \text { trộm } & \left(\mathrm{a}^{4}\right) & \text { voler, to rob } \\ \text { k:456 } & \text { cỏ } & \left(\mathrm{a}^{5}\right) & \text { herbe, grass } \\ \text { la:456 } & \text { lửa } & \left(\mathrm{a}^{5}\right) & \text { feu, fire } \\ \text { la:j456 } & \text { lưỡi } & \left(\mathrm{a}^{6}\right) & \text { langue, tongue } \\ \mathrm{p}^{\mathrm{h}} \text { o:456 } & \text { vỗ } & \left(\mathrm{a}^{6}\right) & \text { frapper, to slap }\end{array}$

\section{Formation d'un système hétérodoxe à 4 tons}

Ces systèmes à 4 tons caractérisent les langues et dialectes du sous groupe hung (pong ou phong, toum, liha) et ceux des dialectes vietnamiens du Centre-Nord localisés dans le bassin du Sông Gianh [Hướng Hóa, Hạ Trạch, Cao Lao Hạ]. La réduction de 6 à 4 du nombre des tons n'est qu'apparente car il ne s'agit pas, comme on pourrait le penser, d'un stade poussé des confusions tonales. L'origine de cette réduction n'a rien a voir avec les réductions aléatoires de 6 à 5 tons attestées dans certains dialectes vietnamiens et mường. Ces systèmes à 4 tons sont trop particuliers pour être l'aboutissement de l'usure d'un système à 6 tons. Il faut donc trouver un autre type d'explication. 
Hypothèse: Deux langues, lexicalement proche parentes, sont en contact; l'une est la langue A dominée, l'autre est la langue B dominante qui se propage sur la première. Nous ne considérerons qu'une seule série tonale car les faits ont dû se produire bien avant les confusions des séries d'initiales . La langue A présente une opposition à trois termes: a (non marqué) $v s \mathrm{a}^{\mathrm{P}}$ (constriction) $v s$ ah (spirante finale), ces caractéristiques qui étaient celles du PVM sont encore assez bien représentées en maleng malgré l'usure de son système. L'autre, la langue dominante $\mathrm{B}$, présente une opposition tonale à trois termes: a (non marqué) vs a['] (modulé + faible constriction ?) vs a['] (forte constriction). Cette situation est celle du cuối chăm avec la bipartition en plus.

\begin{tabular}{|c|c|c|c|c|}
\hline \multirow[t]{2}{*}{ langue $\mathrm{A}$} & $\mathrm{a}$ & $a^{2}$ & $\mathrm{ah}$ & \multirow[t]{2}{*}{ (type maleng) } \\
\hline & \multicolumn{3}{|c|}{$\Uparrow$} & \\
\hline lanoue $B$ & $\mathrm{a}$ & $\mathrm{a}\left[{ }^{\prime}\right]$ & $\mathrm{a}\left[{ }^{\prime}\right]$ & (type cuối chăm) \\
\hline
\end{tabular}

La langue dominante $\mathrm{B}$ influence la prononciation des locuteurs de la langue dominée A par le biais des correspondances lexicales. en gros, les locuteurs de A tendent à imiter, dans la mesure du possible, la prononciation des locuteurs de $\mathrm{B}$. Sous la pression de $\mathrm{B}$, la langue $\mathrm{A}$ tend à remplacer son -h final par le ton constrictif a ['] de B. Mais, comme la langue A n'est pas encore tonale, elle interprète ce ton $\mathrm{a}\left[\right.$ '] de $\mathrm{B}$ par son propre $\mathrm{a}^{\text {? }}$, identification qui va de soi car ces deux articulations sont, sinon les mêmes, du moins très proches, $a^{2}$ n'étant que la notation dans une langue non tonale et a['] celle dans une langue tonale. Donc, globalement, il y a eu le changement $a h>a^{?}$. L'existence en cuối du ton constrictif a ['] est dans l'ordre des choses car on sait par ailleurs que, lors de la tonogénèse, la constriction glottale (pour d'autres creakiness) est la première étape de la transformation de $-\mathrm{h}$ final vers un ton mélodique (Sagart 1988). Quant au ton $\mathrm{a}\left[{ }^{\prime}\right]$ de B, la langue A n'étant pas tonale n'a d'autres moyens que de l'interpréter par son propre $\mathrm{a}^{3}$, identification qui a pu être facilitée par le fait que ce ton a['] était sans doute faiblement constrictif, comme le ton nặng en viet. Globalement la langue A, d'une part a perdu ceux des traits qui n'existaient pas dans la langue B (ah), d'autre part a gardé ceux qui existaient ou étaient identifiables $\left(\mathrm{a}^{\mathrm{P}}\right)$. D'une opposition à trois termes on est passé à une opposition à deux termes avec d'inévitables pertes distinctives. Ce réaménagement de la langue dominée A étant bien établi, le vocabulaire de la langue dominante $\mathrm{B}$, ancêtre du cuối, a pu remplacer par la suite le vocabulaire de la langue dominée en s'adaptant à ce nouveau carcan phonétique. Le résultat en sera la formation de la langue 
ancêtre du sous groupe hung dont les langues filles, le pong et le toum, attestent aujourd'hui un système purement tonal à 4 tons à la suite des confusions des séries d'initiales.

Ce scénario n'est pas une vision de l'esprit. Les langues thổ, dont le cuối, sont localisés dans les régions basses du Nghệ An tandis que les langues hung, dont le pong et le toum, sont localisés dans les régions montagneuses un peu à l'ouest, des deux côtés de la frontière lao-viet. Des considérations lexicales conduisent à penser que la langue ancêtre du sous groupe thổ s'est propagée vers les zones reculées en submergeant une autre langue, proche parente mais restée plus conservatrice, du type maleng ou sách/rục. Les langues du sous groupe hung seraient le résultat de ce déplacement. Ce fait expliquerait la place centrale que le pong occupe dans l'ensemble des langue viet-muong. Isolé au milieu des populations de langue thai-tai et donc, contrairement au cuối, préservé de l'influence constante du vietnamien il a préservé, à peu de chose près, le système vocalique du proto viet-muong (Ferlus 1997a).

Du point de vue de la langue dominante, on pourrait effectivement considérer un système de base à 6 tons qui aurait évolué vers 4 tons, comme d'autres ont évolué vers 5 tons. Il ne semble pas que ce soit le cas car le passage de 6 à 5 tons n'est dû qu'a un phénomène d'usure interne tandis que la passage éventuel de 6 à 4 tons est un phénomène d'interprétation entre des structures différentes, quoique voisines, dans une situation particulière de contact entre deux langues proches parentes. Ce type de système tonal est dit 'hétérodoxe' parce que sa formation ne peut s'expliquer ni par les seules considérations de phonétique historique, ni par des irrégularités des lois, ni par une usure des traits phonétiques.

On a de bonnes raisons de penser que la formation des dialectes vietnamiens à 4 tons procède du même type de phénomène. Dans cette région la langue dominante serait une forme ancienne de vietnamien et la langue dominée une forme de sách/rục dont les derniers représentants sont justement localisés non loin de là dans le haut du fleuve Sông Gianh.

\section{Le cas du maleng brô}

Le maleng brô (Ferlus 1997b), dialecte en voie d'extinction du sousgroupe maleng, est parlé dans le district de Nhommalat, province de Khammouan, au Laos.

- 4 tons dans les syllabes en finale sonore;

- 2 tons dans les syllabes en finale sourde;

- pas de -h final;

- voix soufflée / hauteur mélodique;

- modification d'aperture sous les deux séries. 
Donnons le schéma tonal associant les notations étymologiques et les réalisations phonétiques précisant le souffle et la constriction laryngale:

\begin{tabular}{|c|c|}
\hline$a^{1}[\mathrm{v}]$ & $a^{35}\left[\mathbf{v}^{2}\right]$ \\
\hline$a^{2}[\grave{v}]$ & $a^{46}\left[\grave{v}^{2}\right]$ \\
\hline
\end{tabular} \mid \begin{tabular}{|l|} 
at7 $[\mathrm{vt}]$ \\
\hline
\end{tabular}

Comme le sách/rục et le thavung, le maleng brô est représentatif d'un système à registre de type de voix avec ici une opposition /voix claire tendue/vs /voix soufflée relâchée/ pour toutes les voyelles. En série haute les voyelles sont sensiblement plus ouvertes que leurs homologues de série basse. Les voyelles de série basse sont toutes soufflées, cependant la fermeture par prédiphtongaison ne porte que sur les trois unités les plus ouvertes. Les tons a 35 et a 46 sont nettement constrictifs.

\begin{tabular}{|c|c|c|c|c|c|}
\hline \multicolumn{3}{|c|}{$\begin{array}{c}\text { série haute }\left(\mathrm{a}^{1} \mathrm{a}^{35} \mathrm{a}^{7}\right) \\
\text { voyelles claires tendues }\end{array}$} & \multicolumn{3}{|c|}{$\begin{array}{l}\text { série basse }\left(\mathrm{a}^{2} \mathrm{a}^{4} 6 \mathrm{a}^{8}\right. \\
\text { voy. soufflées relâchées }\end{array}$} \\
\hline I: & $\stackrel{\dot{t}}{\mathrm{t}}:$ & U: & ì: & I: & ù: \\
\hline ę: & $\Lambda:$ & ọ: & è: & ว̀: & f: \\
\hline è: & & æ: & e غ̀ & & oગ̀ \\
\hline
\end{tabular}

L'ancien - $\mathrm{h}$ final (ton attendus $\mathrm{a}^{5}-\mathrm{a}^{6}$ ) s'est confondu dans la constriction - ${ }^{?}$ (tons attendus $\mathrm{a}^{3}-\mathrm{a}^{4}$ ). L'ancien $-\mathrm{s}$ final a subi la même évolution par l'intermédiaire du changement $-\mathrm{s}>-\mathrm{jh}$. Comme en pong et en toum, la chute $\mathrm{du}$ - $\mathrm{h}$ final en maleng brô n'a pas entraîné la formation d'une unité tonale distincte qui aurait permis un transfer d'opposition. La constriction glottale du maleng brô (notée ici $\mathrm{a}^{3}$ ) dont l'articulation est nettement plus marquée que celle du sách/rục et du thavung, résulte donc de la confusion des anciennes laryngales $-\mathrm{h}$ et $-^{\mathrm{P}} \mathrm{du}$ PVM.

Les tons $\mathrm{a}^{1-\mathrm{a}^{2}}$ du maleng brô correspondent globalement aux tons a-à du viet, tandis que les tons a 35 -a 46 correspondent globalement aux tons á-ạ ou ả-ã. Donnons quelques exemples en utilisant le témoignage du maleng proprement dit qui a l'avantage d'avoir préservé la spirante laryngale finale.

\begin{tabular}{|c|c|c|c|c|}
\hline \multicolumn{2}{|c|}{ maleng brô } & maleng & viet & \\
\hline sa:j & (a1) & saij & tai & oreille, ear \\
\hline$k^{\ominus} \mathrm{san}$ & (a1) & $\mathrm{k}^{\circ} \mathrm{san}$ & răng & dent, teeth \\
\hline $\mathrm{s}^{2} \mathrm{rec}:$ & (a1) & $\partial^{\mathrm{h}} \mathrm{rec}:$ & chày & pilon, pestle \\
\hline roj̀ & $\left(a^{2}\right)$ & ${ }^{\mathrm{h}}$ ro: & rùa & tortue, tortoise \\
\hline
\end{tabular}




\begin{tabular}{|c|c|c|c|c|}
\hline \multicolumn{2}{|c|}{ maleng brô } & maleng & viet & \\
\hline $\mathrm{p}^{\curvearrowright}$ lù: & $\left(a^{2}\right)$ & $\mathrm{p}^{\ominus} 1 \mathrm{u}:$ & trầu & bétel, betel-leaf \\
\hline cì:y & $\left(a^{2}\right)$ & si:y & chân & pied, foot \\
\hline $6 æ: j^{2}$ & $\left(a^{35}\right)$ & $6 a: j^{?}$ & muối & sel, salt \\
\hline$c:^{?}$ & $\left(a^{35}\right)$ & $c a i^{2}$ & chó & chien, $d o g$ \\
\hline plę: ${ }^{?}$ & $\left(a^{35}\right)$ & $p^{2} 1 e_{:}{ }^{P}$ & trái & fruit, fruit \\
\hline tæ: ${ }^{2}$ & $\left(a^{35}\right)$ & $\operatorname{tah}$ & đỏ & rouge, red \\
\hline $6 a:^{2}$ & $\left(a^{35}\right)$ & $6 a h$ & mửa & vomir, to vomit \\
\hline$t^{a} p a:^{2}$ & $\left(a^{35}\right)$ & $\mathrm{t}^{\ominus} \mathrm{pah}$ & vả & giffler, to slap \\
\hline $\mathrm{k}^{\ominus} \mathrm{rìm} \mathrm{m}^{?}$ & $\left(a^{46}\right)$ & $\mathrm{k}^{\partial} \mathrm{rim}^{2}$ & sấm & tonnerre, thunder \\
\hline $\mathrm{k}^{\ominus} \mathrm{j} \mathrm{i} \mathrm{r}^{?}$ & $\left(a^{46}\right)$ & $\mathrm{jij} 1^{2}$ & dậy & se réveiller, to awake \\
\hline$m^{2} \eta \partial ̀:^{?}$ & $(\mathrm{a} 46)$ & $m^{\curvearrowright}$ yว: ${ }^{?}$ & ngựa & cheval, horse \\
\hline $\mathrm{k}^{\circ}$ roj $^{?}$ & $\left(a^{46}\right)$ & $\mathrm{k}^{\circ} \mathrm{roh}$ & sủa & aboyer, to bark \\
\hline mù:j ${ }^{?}$ & $\left(a^{46}\right)$ & mujh & mũi & nez, nose \\
\hline$k u_{: j}{ }^{?}$ & $\left(a^{46}\right)$ & kujh & củi & $\begin{array}{l}\text { feu/bois de chauffe, } \\
\text { fire/firewood }\end{array}$ \\
\hline
\end{tabular}

Le maleng brô appartient bien à la catégorie des systèmes tonals hétérodoxes à 4 tons, à ceci près qu'ici les caractéristiques tonales sont augmentées de traits phonétiques, voix soufflée et constriction glottale, considérés parfois comme des caractéristiques de langues à registres de type de voix. Autre différence, la génèse du système tonal du maleng brô ne semble pas devoir s'expliquer par les mêmes causes que celles envisagées dans le cas du pong, $\mathrm{du}$ toum et des dialectes viet du Centre-Nord. La perte du -h final est vraisemblablement due à l'influence du lao, langue dans laquelle cette finale est justement absente.

\section{Considérations générales sur la tonogénèse}

Le deuxième stade de la tonogénèse est un phénomène aujourd'hui bien compris. Les confusions de séries d'initiales et leurs multiples conséquences n'ont plus de secret pour les linguistes en diachronie. Ces processus continuent de se propager et sont actuellement observables dans certaines langues de l'Asie du Sud-Est. Le premier stade de la tonogénèse, celui qui implique les laryngales finales $(-?-h)$, quoique globalement bien cerné, réserve encore quelques points obscurs dans son déroulement. Précisons que cette hiérarchisation en 'premier stade' et 'deuxième stade' ne fait que traduire la situation la plus générale. Localement il est possible d'observer des langues qui commencent par le deuxième stade, ou même qui ne connaissent que celui-ci.

La transphonologisation des laryngales finales en unités tonales doit d'abord être resituée dans l'évolution générale des unités finales de syllabe principale. Soit le système des finales, le plus complet possible, observable 
dans quelques langues austroasiatiques conservatrices comme le khamou du Nord-Laos par exemple.

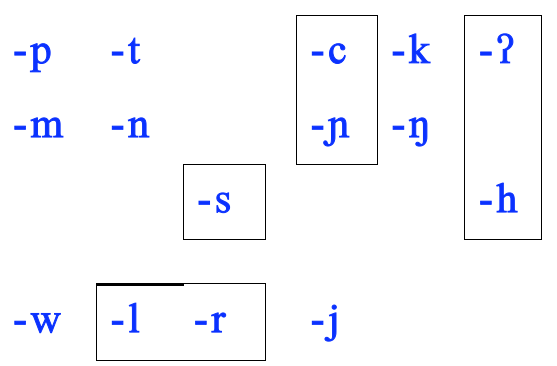

Dans la famille austroasiatique on peut observer, selon les langues, une tendance générale à simplifier ce système, les unités les plus menacées étant les laryngales $(-\mathrm{P}-\mathrm{h})$, les palatales $(-\mathrm{c}-\mathrm{n})$, la sifflante $(-\mathrm{s})$ et les liquides $(-1$ $-r)$. De toutes les unités de ce système les laryngales sont les seules dont l'articulation ne fait intervenir que la glotte, plus exactement les muscles et les parois de la glotte. Or les manifestations de variation mélodique, intonation ou ton, eux aussi ne font intervenir que ces mêmes organes. Il n'est donc pas surprenant qu'une modification des laryngales finales, amuïssement suivi de chute, entraîne l'apparition de traits mélodiques. Cette condition n'est cependant pas suffisante comme nous allons le voir dans ce qui suit.

Dans la structure originelle des langues austroasiatiques la syllabe principale était toujours fermée par une consonne, en un mot il n'y avait pas de syllabe ouverte (finale Ø). Les travaux sur le môn le montrent clairement (Diffloth 1984, Ferlus 1983). Cette langue qui a, en particulier, préservé jusqu'à aujourd'hui l'occlusive glottale finale du proto môn reflète assez bien de ce point de vue l'état ancien de l'austroasiatique. Le khmer en revanche a perdu l'occlusive glottale finale, vraisemblablement aux alentours du milieu du premier millénaire de notre ère (Ferlus 1992), sans pour autant développer une marque tonale. Les syllabes en $-?$ du môn correspondent donc à des syllabes ouvertes en khmer moderne. Donnons quelques exemples de correspondances actuelles entre les deux langues.

\begin{tabular}{|c|c|c|}
\hline môn & khmer & \\
\hline toe? & dei & terre, earth \\
\hline pəe? & 6ei & trois, three \\
\hline $\mathrm{p}^{\mathrm{h}} \mathrm{e:}$ ? & $p^{h}$ è: & loutre, otter \\
\hline $\operatorname{ta} ?$ & ta: & père/grand-père, father/grandfather \\
\hline$c^{\mathrm{h}} \mathrm{u} ?$ & $c^{\mathrm{h}} \grave{\partial}:$ & arbre, tree \\
\hline sa? & sro:v & paddy, unhusked rice \\
\hline
\end{tabular}


N'ayant pas de syllabe ouverte, conformément au modèle austroasiatique, le khmer ancien a pu perdre son occlusive glottale finale sans perte conséquente de trait distinctif et donc sans risque de confusion lexicale. Le développement d'une marque tonale pour assurer une éventuelle transphonologisation n'était pas nécéssaire. Donc, comme on peut le voir, pour comprendre les phénomènes de la tonogénèse il faut tenir compte à la fois de la situation phonétique et des contraintes phonologiques.

Le PVM, contrairement au proto khmer et au proto môn, possédait des syllabes en voyelle ouverte (finale $\varnothing$ ) à côté des syllabes en laryngale finale (finale $-?-\mathrm{h}$ ) d'où la triade des rimes a $\mathrm{a}^{2} \mathrm{ah}$, formes prises par ces trois types de syllabes dans la phase tardive du PVM juste avant les premières manifestations tonales. Dans ce contexte, la chute pure et simple de la constriction glottale risquait d'entraîner une perte de distinction par la confusion éventuelle des rimes a et $\mathrm{a}^{2}$. Les langues VM ont réagit en créant un premier ton marqué, actuellement $\mathrm{a}^{3}-\mathrm{a}^{4}$ (viet: á-ạ), dès lors opposable à un ton non marqué, actuellement $\mathrm{a}^{1-\mathrm{a}^{2}}$ (viet: a-à). C'est le stade des langues, mis à part la confusion des séries d'initiales, qui ont préservé le -h final comme le sách/rục, le thavung et le maleng. La place de $\mathrm{a}^{2}$ étant libre, ah pouvait évoluer vers une constriction puis vers un ton mélodique, actuellement $\mathrm{a}^{5-\mathrm{a}^{6}}$ (viet: ả-ã). Nous sommes dans la situation des langues à 6 tons de base comme le vietnamien, le mường, le nguồn et le cuối. Le passage par un stade constrictif, qui est encore celui du cuối, peut très bien s'expliquer par des contraintes d'économie articulatoire, une constriction consommant moins de flux expiratoire qu'une spirante.

L'existence de syllabes ouvertes en PVM, contrairement au proto khmer et au proto môn, est un des problèmes les plus intrigants du comparatisme austroasiatique. Le môn moderne possède des mots non empruntés en syllabe ouverte mais leur origine est parfaitement connue. Ils se sont formés à la suite de la chute des liquides finales (-1 -r) en môn moyen. Le cas des langues VM est bien différent car il est difficile d'y expliquer l'origine des mots en syllabe ouverte. Il a été bien établi que des mots PVM en rime $\mathrm{a}^{\mathrm{P}}$ (viet: á-ạ) correspondent à des mots fermés par - 2 en khamou; cette dernière langue, proche géographiquement et génétiquement du VM, est prise ici comme représentant exemplaire de l'austroasiatique. C'est d'ailleurs ce type de correspondance qui avait conduit Haudricourt (1954) à formuler son hypothèse sur l'origine des tons en vietnamien. 


\begin{tabular}{|c|c|c|c|}
\hline khamou & PVM & viet & \\
\hline so? & $* \cos { }^{2}$ & chó & chien, $d o g$ \\
\hline ka? & ${ }^{*} \mathrm{ka}_{\mathrm{i}}{ }^{2}$ & cá & poisson, fish \\
\hline ple? & ${ }^{*} \mathrm{p}-1 \mathrm{e}_{\mathrm{i}}^{\mathrm{P}}$ & trái & fruit, fruit \\
\hline ryko? & ${ }^{*} \mathrm{r}-\mathrm{ko} \mathrm{i}^{\mathrm{P}}$ & gạo & riz décortiqué, husked rice \\
\hline klna? & $*_{\text {na: }}{ }^{2}$ & nhựa & résine, glu, resin, birdlime \\
\hline
\end{tabular}

Toutefois, il y a de nombreux exemples où des mots PVM en rime a (viet: a-à) correspondent également à des mots fermés par - 1 en khamou.

\begin{tabular}{|c|c|c|c|}
\hline khamou & PVM & viet & \\
\hline $\mathrm{kma?}$ & ${ }^{*} \mathrm{k}-\mathrm{ma}$ & mưa & pluie, rain \\
\hline mpo? & $* \mathrm{k}-\mathrm{po}$ & bao & rêver, to dream \\
\hline Jru? & *c-ru: & sâu & profond, deep \\
\hline yi? & *yi: & ngày & jour, day \\
\hline mbo? & *bo: & bò & zébu, zebu \\
\hline
\end{tabular}

Le fait surprenant et qui pose problème n'est pas tant l'existence de l'un ou de l'autre de ces deux types de correspondance, khamou $-?$ avec viet a-à ou á-ạ, mais leur présence simultanée dans un même état de langue. Le problème de cette double correspondance, et de ses implications sur la conception de la tonogénèse du vietnamien ont été soulignés par ${ }^{\text {? }}$. Gage (1985) et par G. Diffloth (1989). Des explications ont été proposées mais aucune n'est pleinement satisfaisante. Quoiqu'il en soit, ce problème ne sera pas abordé ici pour les raisons qu'il n'affecte pas le déroulement de la tonogénèse dans les langues VM et qu'il concerne plutôt leur rapport avec les autres groupes de l'austroasiatique.

Tout au long de cette étude nous avons exposé et analysé les solutions les plus diverses et les plus représentatives de la tonogénèse viet-muong à l'aide d'exemples judicieusement choisis dans l'éventail des possibilités. Ces phénomènes qui font intervenir des mécanismes articulatoires élémentaires et universels ont une portée qui dépasse largement le cadre restreint du champ d'étude et contribuent positivement à l'enrichissement de la typologie des changements phonétiques. L'expérience viet-muong a ceci de particulier qu'elle permet de saisir les phénomènes au plus près de leur commencement, elle est donc utilisable avec profit dans d'autres aires de familles linguistiques comme le sino-tibétain, le tai-kadai ou le miao-yao où ces mêmes phénomènes sont plus avancés dans leur évolution. 
Adresse professionnelle

Michel Ferlus

EHESS, Centre de Recherches

Linguistiques sur l'Asie Orientale,

Unité Associée au CNRS \# 1025

54, bl Raspail, 75006 Paris, France
Adresse personnelle:

Michel Ferlus

72 rue Grande

47440 Casseneuil

France

<jrmferlus@wanadoo.fr>

\section{RÉFÉRENCES}

Cadière, Père Léopold. 1902. Phonétique annamite (dialecte du HautAnnam). (= Publication de l'Ecole Française d'Extrême-Orient, 3) Paris: Ernest Leroux.

Đặng Ngiêm Vạn, Chu Thái Sơn, Lưu Hùng. 1986. Les ethnies minoritaires du Vietnam. Hanôi: Éditions en langues étrangères.

Diffloth, Gérard. 1989. "Proto-Austroasiatic creaky Voice". Mon-Khmer Studies 15. 139-54.

------. 1984. The Dvaravati Old Mon Language and Nyah Kur. Bangkok: Chulalongkorn University Printing House.

Ferlus, Michel. 1979. "Lexique thavung-français". Cahiers de Linguistique Asie Orientale 5. 71-94.

--------. 1982. "Spirantisation des obstruantes médiales et formation du système consonantique du vietnamien". Cahiers de Linguistique Asie Orientale 11:1. 83-106.

12: $1-90$.

1983. "Essai de phonétique historique du môn". Mon-Khmer Studies

1991. "Le dialecte vietnamien de Vinh". Présentation faite à l'occasion de la 24th International Conference on Sino-Tibetan Languages and Linguistics, Bangkok, 7-9 oct. 1991/ Chiang Mai, 10-11 oct. 1991.

1992. "Essai de phonétique historique du khmer (Du milieu du premier millénaire de notre ère à l'époque actuelle)". Mon-Khmer Studies 21. 57-89.

1994. "Quelques particularités du cuối chăm, une langue viet-muong du Nghệ An (Vietnam)". Présentation faite à l'occasion des Neuvièmes journées de linguistique de l'Asie Orientale, EHESS/CNRS, Paris 5-6 mai 1994.

--------. 1995. "Particularités du dialecte de Cao Lao Hạ (Quảng Bình, Vietnam)". Présentation faite à l'occasion des Dixièmes journées de linguistique de l'Asie Orientale. EHESS/CNRS, Paris, 16-17 mai 1995.

--------. 1996. "Langues et peuples viet-muong". Mon-Khmer Studies 26: 7-28.

-------. 1997a. "Problèmes de la formation du système vocalique du vietnamien". Cahiers de Linguistique Asie Orientale 26:1. 37-51 (Publication de "Formation du système vocalique du vietnamien", présenté à la 27th Inter- 
national Conference on Sino-Tibetan Languages and Linguistics, CNRS, Paris/Sèvres, 12-16 octobre 1994.)

------. 1997b. "Le maleng brô et le vietnamien". Mon-Khmer Studies, 27. 55-66.

Gage, William W. 1985. "Glottal stops and Vietnamese tonogenesis". For Gordon F. Fairbanks ed. by V. Z. Acson \& R. L. Leed (= Oceanic Special Publication, 20), 21-31. Honolulu: University of Hawaii Press.

Haudricourt, André Georges. 1954. "De l'origine des tons en vietnamien". Journal Asiatique 242. 69-82.

Henderson, Eugénie J. A. 1952. "The main features of Cambodian pronounciation". Bulletin of the School of Oriental and African Studies 14. 149-74.

Nguyễn Phú Phong, Trần Trí Dõi, Michel Ferlus. 1988. Lexique vietnamienruc-français. Paris: Sudestasie.

Nguyễn Tài Cẩn. 1995. Giáo trình lịch sủ ngũ âm tiếng Việt, [Cours de phonétique historique de la langue vietnamienne]. Hànội: Nhà xuất giáo.

Nguyễn Văn Lợi. 1993. Tiếng rục [La langue rục]. Hànội: Nhà xuất bản khoa học xã hội.

Rhodes, Père Alexandre de. 1651. Dictionarium Annamiticum Lusitanum et Latinum. Rome. Reproduit et augmenté d'un dictionnaire traduisant les mots et expressions en vietnamien moderne: Tù Điển Annam-Lusitan-Latin, 1991, Hồ Chí Minh ville.

Sagart, Laurent. 1988. "Glottalised tones in China and South-East Asia". Prosodic Analysis and Asian Linguistics: To honour R.K. Sprigg ed. by David Bradley, Eugénie J. A. Henderson \& Martine Mazaudon (= Pacific Linguistics, C-104), 83-93. Canberra: Canberra, Department of Linguistics, Research School of Pacific Studies, Australian National University.

Sokolovskaia N.K., Nguyễn Văn Tài. 1987. La langue mương: Matériaux de l'expédition linguistique Sovieto-Vietnamienne de 1979. Moscou: Éditions scientifiques. [En russe].

\section{SUMMARY}

The Viet-Muong languages present a wide variety of systems having four to six tones, and reflecting two earlier systems: a six-tone system and a fourtone system in which final $-\mathrm{h}$ is preserved. Vietnamese, Mường and Cuối are representative of a pure six-tone system in the traditional definition. Sách/Rục and Thavung have a four-tone system in which vowel breathiness is preserved in the low tone series. When in such a system breathiness is lost too fast, as in Maleng, the result is a kind of eroded two-tone system. In some languages like Pong and some Vietnamese dialects, final $-\mathrm{h}$ was lost without giving contrastive tones, the result being a kind of heterodox four-tone system. The situation of tone systems in Viet-Muong languages is of the highest importance for the theory of tonogenesis. 


\section{RÉSUMÉ}

Les langues viet-muong offrent un large éventail de systèmes tonals, de quatre à six tons, provenant tous de deux systèmes antérieurs de base: l'un à six tons et l'autre à quatre tons où le $-\mathrm{h}$ est préservé. Le vietnamien, le mường et le cuối sont représentatifs d'un pur système à six tons dans leur définition la plus classique. Le sách/rục et le thavung ont un système à quatre tons avec des voyelles soufflées sous les tons de la série basse. La chute trop rapide du caractère soufflé, comme en maleng, donne une sorte de système érrodé à deux tons. En pong et dans certains dialectes vietnamiens le - $\mathrm{h}$ final est tombé sans entraîner la création de tons supplémentaires, le résultat étant la formations de systèmes tonals hétérodoxe à quatre tons. La variété des systèmes tonals en viet-muong représente un apport important à une théorie de la tonogénèse.

\section{ZUSAMMENFASSUNG}

Die Viet-Muong-Sprachen bieten ein breites Spektrum von Systemen zwischen vier und sechs Tönen; so stellen sie beide Entwicklungen zweier älterer Systeme, und zwar ein 6-Tonsystem und ein 4-Tonsystem, in welchen Auslaut - $\mathrm{h}$ erhalten geblieben ist. Vietnamesisch, Mường and Cuối sind repräsentativ für ein reines 6-Tonsystem nach herkömmlicher Definition. Sách/Rục und Thavung hingegen besitzen ein 4-Tonsystem, in welchem eine rauhe Vokalbehauchung in der unteren Tonreihe erhalten geblieben ist. Wenn in einem solchen System die rauhe Behauchung zu schnell verlorengeht, wie im Maleng, ist das Ergebnis eine Art erodiertes 2-Tonsystem. Im Pong und einigen vietnamesischen Dialekten ist Auslaut - $\mathrm{h}$ verlorengegangen, ohne dass sich jedoch enstprechende kontrastierende Töne entwickelten: das Ergebnis ist eine Art heterodoxes 4-Tonsystem. Die Vielfalt der Tonsysteme in den VietMuong-Sprachen ist von grosser Bedeutung für die Theorie der Tonogenese.

février 2007 : mise en unicode, correction de quelques fautes, correction du résumé en allemand 


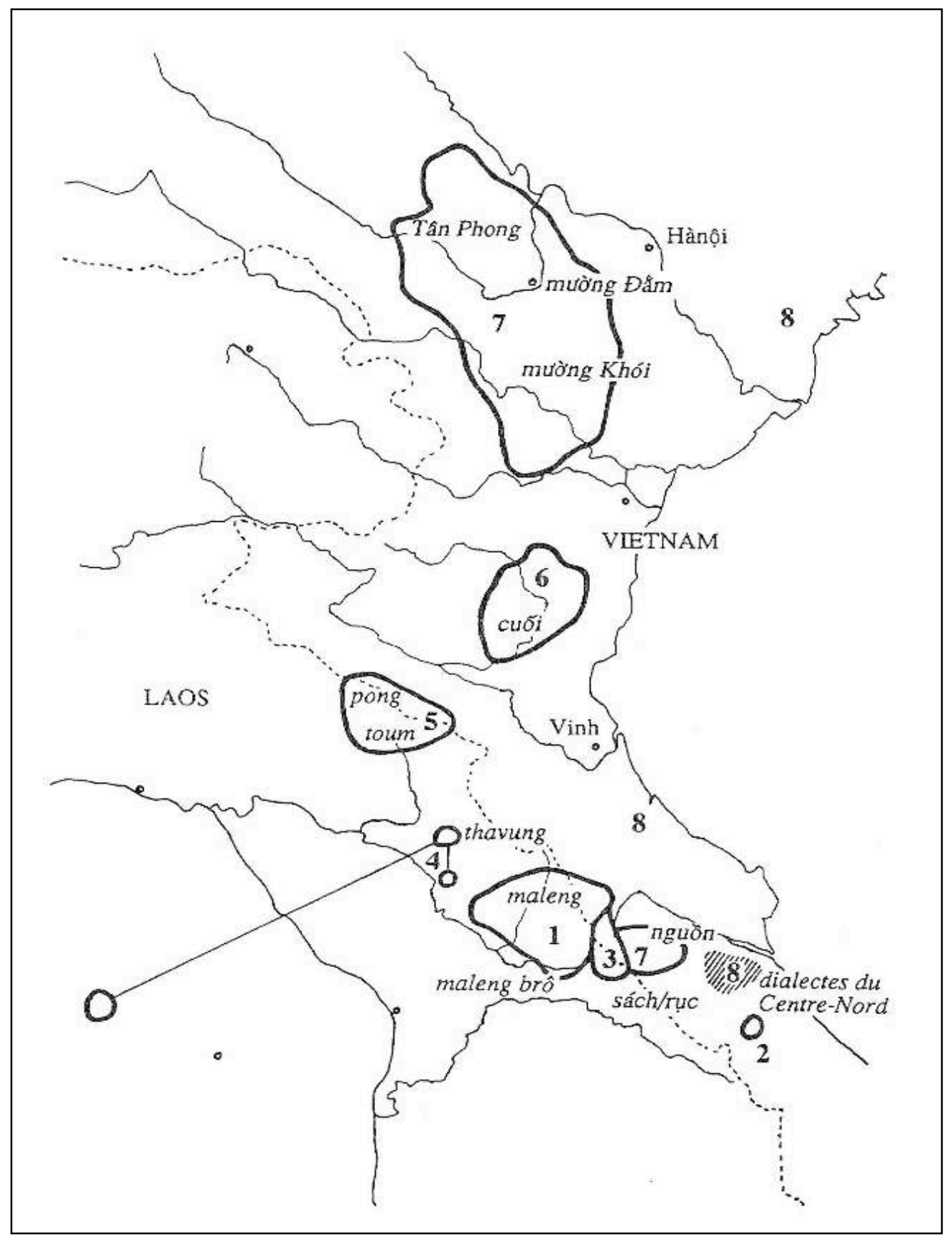

Carte des langues et dialectes viet-muong

(La carte ne mentionne que les numéros des huit sous groupes et les noms des langues et dialectes utilisés.) 